\title{
Producción y tasa de descomposición de hojarasca en áreas bajo rehabilitación en El Porvenir, Hidalgo, México
}

\author{
Production and decomposition rate of leaf litter in areas under \\ rehabilitation in El Porvenir, Hidalgo, Mexico
}

\author{
María Tonatzín García-Osorio', Francisca O. Plascencia-Escalante ${ }^{*}$, Gregorio Ángeles-Pérez', \\ Francisco Montoya-Reyes ${ }^{2}$ y Leonardo Beltrán-Rodríguez ${ }^{3}$
}

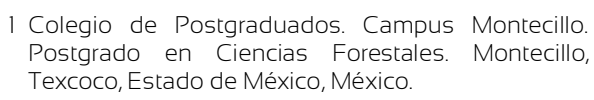

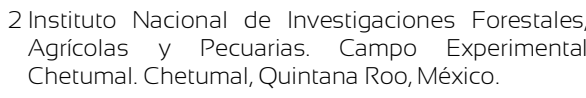

\author{
3 Universidad Nacional Autónoma de México. \\ Instituto de Biología. Jardín Botánico. Laboratorio de \\ Etnobotánica Ecológica. Ciudad de México, México
}

* Autora de correspondencia. fplascencia@colpos.mx

\section{RESUMEN}

La producción de hojarasca es la fuente principal de materia orgánica en los ecosistemas forestales y el análisis del balance entre esta y su descomposición permite comprender la dinámica nutrimental y su aporte en el crecimiento vegetal. El objetivo del presente trabajo fue determinar el efecto de la edad de reforestación sobre la producción y tasa de descomposición de hojarasca en El Porvenir, Hidalgo, México. Se seleccionaron tres condiciones (R5, R12 y R14) con diferentes edades de reforestación (5 años, 12 años y 14 años, respectivamente), más un sitio de referencia (SR) con remanentes de vegetación natural. En cada condición se seleccionaron al azar 10 puntos de muestreo donde se registró la producción mensual de hojarasca en trampas de $0.5 \mathrm{~m}^{2}$ durante un año. Se establecieron cuatro lotes por condición para determinar la tasa de descomposición de hojarasca y los contenidos de $\mathrm{C}$ y N. La mayor producción de hojarasca se registró en R14 $\left(0.3099 \mathrm{~kg} \mathrm{~m}^{-2}\right.$ año-1), mientras que la menor en R5 $\left(0.0436 \mathrm{~kg} \mathrm{~m}^{-2}\right.$ año-1). Las tasas de descomposición más alta y más baja se registraron en SR $(k=0.676)$ y R14 $(k=0.229)$, respectivamente, con diferencias altamente significativas $(\mathrm{p} \leq 0.0098)$. Al final del periodo de incubación, la mayor concentración de C se presentó en R12 y la de N en R14, mientras que la mayor liberación de ambos compuestos se obtuvo en SR. Se concluye que la edad de la plantación tiene un efecto positivo en la producción de hojarasca, pero no en la tasa de descomposición.

Palabras clave: liberación de carbono y nitrógeno, Pinus cembroides, Pinus greggii, reciclaje de nutrientes, reforestaciones, sitio de referencia.

\section{ABSTRACT}

The leaf litter from trees is the main route of entry of organic matter and its subsequent decomposition allows the flow of nutrients in the ecosystem. The study of these processes in ecosystems under rehabilitation is of vital importance to determine how such actions are influencing their recovery. This study was carried out to determine the effect of reforestations on litter production and decomposition at El Porvenir, Hidalgo State, México. We selected four different conditions: three correspond to areas with different reforestation ages, 5, 12 and 14-year-old (R5, R12 and R14) while the fourth is a reference site (SR) with remnants of natural vegetation. For each condition, 10 sampling points were selected in which litter traps were placed to quantify the litter production for one year. In 4 sampling points per condition we placed litter bags to measure the rate of decomposition for a 12-month period. The reforestation age showed a positive effect of litter production. The largest litter production was recorded in R14 (0.3099 $\mathrm{kg} \mathrm{m}^{-2}$ year $\left.^{-1}\right)$ while the lowest was for R5 (0.0436 $\left.\mathrm{kg} \mathrm{m}^{-2} \mathrm{yr}^{-1}\right)$. The highest and lowest decomposition rates correspond to SR $(k=0.676)$ and $\mathrm{R} 14(k=0.229)$. On the other hand, the increased release of $\mathrm{C}$ was presented in SR and the lowest in R12. For its part, the release of more $\mathrm{N}$ at the end of the incubation period was recorded in SR and the lowest in R14.

KEYWORDS: carbon and nitrogen release, Pinus cembroides, Pinus greggii, nutrient recycling, reforestations, reference site. 


\section{INTRODUCCIÓN}

La producción y descomposición de hojarasca son procesos clave en el funcionamiento de los ecosistemas terrestres (Karberg, Scott y Giardina, 2008; Muoghalu y Odiwe, 2011), debido a que están directamente relacionados con la fotosíntesis y la respiración microbiana, y desempeñan un papel importante en el ciclo global del carbono (Hanpattanakit y Chidthaisong, 2012). La hojarasca proveniente de los árboles, entendida como todas las estructuras reproductivas y vegetativas de las especies que se distribuyen en los ecosistemas (Bisht, Nautiyal, Kuniyal, Prasad y Sundriyal, 2014; Krishna y Mohan, 2017), es la vía principal de entrada de materia orgánica al suelo (Karberg et al., 2008; Chenu, Rumpel y Lehmann, 2015; Krishna y Mohan, 2017).

La descomposición y liberación de nutrientes de la hojarasca a través de una serie de procesos fisicoquímicos, son fundamentales para garantizar el adecuado funcionamiento de los ciclos biogeoquímicos y, con ello, favorecer apropiadas condiciones físicas, químicas y biológicas de los suelos (Castellanos-Barliza y León, 2010). La tasa de descomposición de la hojarasca es controlada por tres factores principales: la temperatura, la humedad y la calidad de la hojarasca (Karberg et al., 2008).

La cantidad y naturaleza de la hojarasca tienen una importante relación con la formación del suelo y el mantenimiento de su fertilidad, de ahí que tanto la cuantificación de su producción como su composición son importantes para comprender los ciclos de los nutrimentos en los bosques (López-Hernández et al., 2013; Bisht et al., 2014; Moreno et al., 2018). Por lo tanto, el estudio de estos procesos en ecosistemas en vías de recuperación es de vital importancia para conocer cómo es que las acciones emprendidas están influyendo en ellos (Rocha-Loredo y Ramírez-Marcial, 2009; Castellanos-Barliza y León, 2010; García-Velásquez, Ríos-Quintana y Molina-Rico, 2010; Riviera et al., 2013; Gaspar-Santos, González-Espinosa, Ramírez-Marcial y Álvarez-Solís, 2015).

Algunos estudios desarrollados en Latinoamérica y el mundo han destacado la importancia de comprender integralmente el proceso de producción y descomposición de la hojarasca en los ecosistemas, mediante el cual los compuestos orgánicos complejos se degradan continuamente en sustancias más simples, liberando nutrientes como subproducto de su descomposición (Yan et al., 2004; Wang, Wang, Xu y Fan, 2010; Rubiano-Cardona, ArcilaCardona, Jiménez-Carmona y Armbrecht, 2013; Bisht et al., 2014; Krishna y Mohan, 2017). Rocha-Loredo y Ramírez Marcial (2009) cuantificaron la producción anual de hojarasca en comunidades sucesionales en dos localidades del estado de Chiapas, y encontraron diferencias significativas entre las condiciones estudiadas, con máxima acumulación de hojarasca en el bosque maduro de pinoencino y menor aporte anual en los sitios con bosque secundario.

En el Parque Nacional Lore Lindu, Sulawesi Central, Indonesia, Triadiati et al. (2011) evaluaron cómo el cambio del uso de la tierra del bosque natural a la agroforestería de cacao influye en la producción, descomposición y liberación de nutrientes en la hojarasca, encontrando que el bosque natural presenta mayor producción anual, mayor tasa de descomposición y libera la mayor cantidad de nitrógeno. Por el contrario, Xuluc-Tolosa, Vestera, Ramírez-Marcial, Castellanos-Albores y Lawrence (2003) encontraron que la tasa de descomposición de la hojarasca está relacionada con la edad de desarrollo forestal en un bosque seco en Campeche, México. Resultado que fue reforzado por los estudios de Zhou et al. (2007) en Guangdong, China, quienes demostraron que la producción de hojarasca está estrechamente relacionada con las etapas sucesionales en bosques reforestados hace 50 años aproximadamente, comparados con comunidades clímax de entre 100 años y 300 años de edad, con una mayor producción en las comunidades conservadas respecto de las etapas pioneras.

En la región de El Porvenir, Hidalgo, México, se establecieron reforestaciones con la finalidad de recuperar ecosistemas afectados por actividades antropogénicas, mismas que impactaron considerablemente a los bosques naturales (Domínguez-Calleros, Rodríguez-Laguna, Capulín-Grande, Razo-Zárate y Díaz-Vásquez, 2017; 
Ventura-Ríos, Plascencia-Escalante, Hernández de la Rosa, Ángeles-Pérez y Aldrete, 2017). La pérdida de la cubierta vegetal trajo consigo problemas de erosión del suelo y modificaciones en la dinámica del reciclaje de nutrientes en el ecosistema (Comisión Nacional Forestal [Conafor, 2012). En 1998 se realizaron las primeras acciones de rehabilitación forestal exclusivamente con reforestaciones en el área, estableciendo como principal especie a Pinus greggii Engelm. ex Parl. (Domínguez et al., 2017; Ventura-Ríos et al., 2017; Montoya-Reyes et al., 2019).

A pesar de la importancia que tienen los procesos de producción y descomposición de hojarasca para el funcionamiento ecosistémico, en esta región son pocas las investigaciones que se han enfocado en evaluar alguno de estos aspectos. Por ejemplo, Domínguez et al. (2017) determinaron que la pendiente de las superficies en donde se realizaron las reforestaciones está estrechamente relacionada con las dimensiones de los árboles plantados y, por lo tanto, con la cobertura de copa, parámetro alométrico a su vez asociado con la cantidad de hojarasca producida. No obstante, estudios de estimacióncomparación entre rodales naturales vs áreas bajo rehabilitación forestal a diferentes edades en la zona de estudio son nulos. Las relaciones entre las tasas de producción y descomposición en el medio rehabilitado son vitales para evaluar la eficacia de este tipo de estrategias y conocer el ciclo de reciclaje de nutrientes de los ecosistemas (Carnevale y Lewis, 2001).

\section{Objetivos}

Determinar el efecto de la edad de la reforestación (R5, R12 y R14) sobre la producción de hojarasca y su tasa de descomposición en el Porvenir, Hidalgo, México. Se espera que las acciones de rehabilitación forestal influyan en los parámetros estudiados entre condiciones, de manera que habría una tasa de producción y descomposición gradual con la edad de rehabilitación, con valores superiores en el sitio de referencia.

\section{MATERIALES Y MÉTODOS}

\section{Área de estudio}

El estudio se llevó a cabo en la localidad de "El Porvenir", municipio de Santiago de Anaya, en el Estado de Hidalgo, dentro de las coordenadas geográficas $20^{\circ} 28^{\prime} 16^{\prime \prime}$ - $20^{\circ} 26^{\prime} 14^{\prime \prime}$ latitud N y $98^{\circ} 58^{\prime}$ 24" - $98^{\circ} 56^{\prime} 17^{\prime \prime}$ longitud $\mathrm{O}$, y las cotas altitudinales de $1800 \mathrm{~m}$ a $2580 \mathrm{~m}$ s.n.m. (Fig. 1). La temperatura media anual es de $15.3{ }^{\circ} \mathrm{C}$, mientras que la precipitación media anual es de $555.6 \mathrm{~mm}$ (Servicio Meteorológico Nacional, 2019). La vegetación natural corresponde a remanentes aislados dominados por Pinus cembroides Zucc. Existen en la región sitios que han sido plantados con especies exóticas y nativas (Casuarina sp., Cupressus sp., Eucalyptus sp., P. cembroides, Pinus patula Schiede ex Schltdl. \& Cham. Pinus pseudostrobus Lindl.), utilizando como principal especie a Pinus greggii Engelm. ex Parl. (Domínguez et al., 2017; Ventura-Ríos et al., 2017; Montoya-Reyes et al., 2019).

Con base en investigaciones previas desarrolladas en el área de estudio (Domínguez et al., 2017; VenturaRíos et al., 2017; Montoya-Reyes et al., 2019), se seleccionaron cuatro condiciones para el desarrollo de la investigación. Tres áreas corresponden a rodales con diferente edad de rehabilitación forestal con P. greggii: 5 años (R5), 12 años (R12) y 14 años (R14); así como un sitio de referencia (SR), que refiere a un bosque natural con presencia de P. cembroides y Juniperus flaccida Schltdl, ubicado a $2 \mathrm{~km}$ a la redonda de las áreas elegidas con rehabilitación forestal y con características alométricas propias de un rodal maduro, no sujeto a manejo forestal (Montoya-Reyes et al., 2019). En cada una de las cuatro condiciones, se establecieron al azar 10 puntos de muestreo en los que se midió la producción de hojarasca, dentro de los cuales se eligieron cuatro sitios también por condición para medir descomposición de acículas (Fig. 1). 

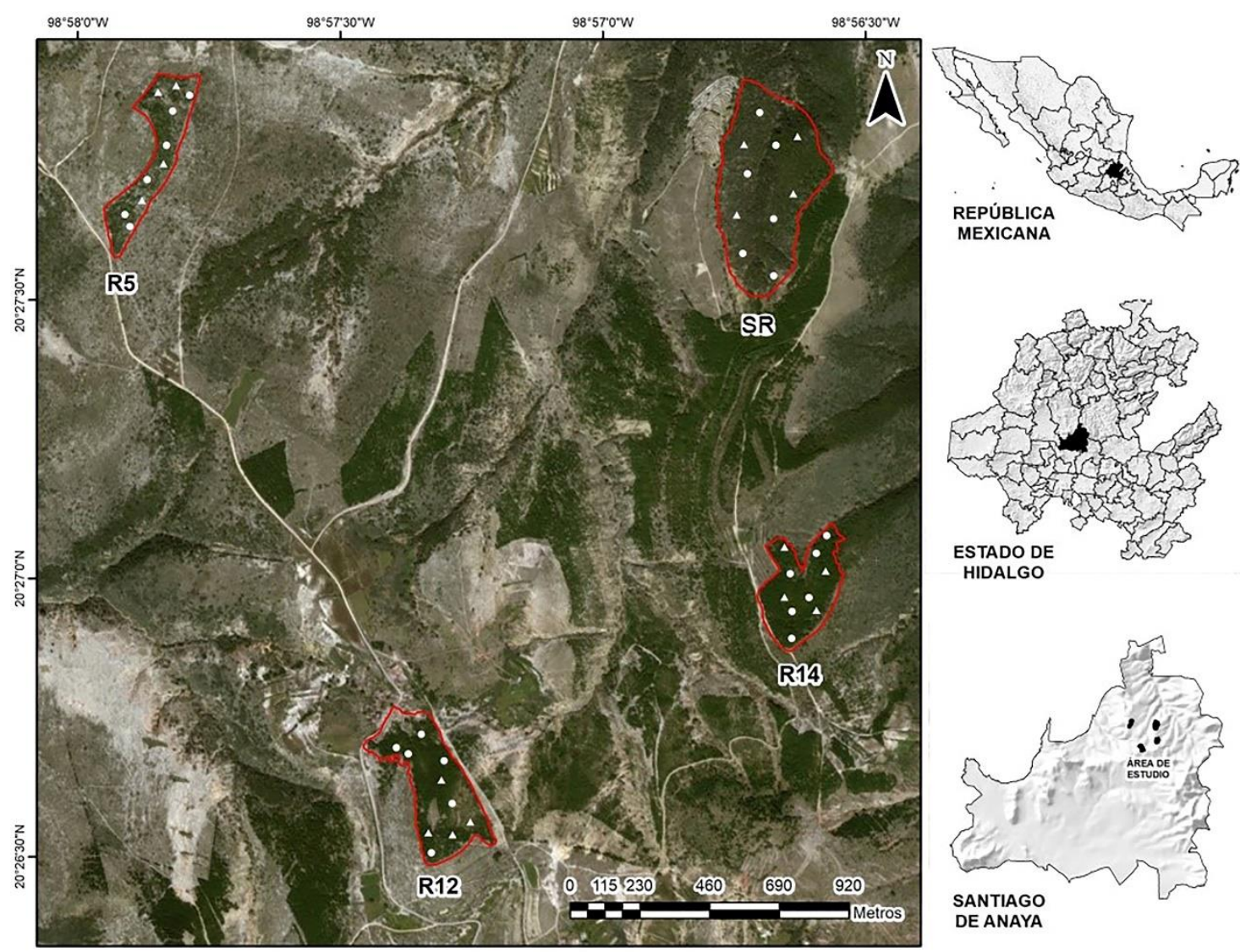

FIGURA 1. Localización del área de estudio.

Se aprecia la condición de las reforestaciones (R5, R12, R14) y el sitio de referencia (SR). Al interior se presentan los pun tos de muestreo establecidos por condición y se identifica con un triángulo aquellos sitios de muestreo en los que adicionalmente se colocaron lotes de descomposición de hojarasca.

\section{Producción de hojarasca}

Para estimar la cantidad de hojarasca que ingresa al sistema, se establecieron de manera aleatoria 10 trampas de hojarasca con forma circular por condición, con un área de colecta de $0.5 \mathrm{~m}^{2}$ cada una, colocadas a $1 \mathrm{~m}$ sobre el nivel del suelo. El material interceptado se colectó cada 30 días (González-Rodríguez et al., 2011) durante un periodo de 12 meses, a partir de febrero del 2015 y hasta enero del 2016.

El material colectado se secó en una estufa de circulación forzada de aire (marca Shel Lab modelo s/070906004) a una temperatura de $70{ }^{\circ} \mathrm{C}$, en las instalaciones del laboratorio de Ciencias Forestales del Colegio de Postgraduados Campus Montecillo, hasta obtener peso constante (Krishna y Mohan, 2017).

\section{Tasa de descomposición}

Para calcular la tasa de descomposición de las acículas se utilizó el método de bolsas de descomposición (Coleman y Crossley, 2003; Graca, Barlocher y Gessner, 2005), elaboradas de malla sombra $(30 \mathrm{~cm} \times 30 \mathrm{~cm})$ con una abertura de $2 \mathrm{~mm}$. Se establecieron de manera aleatoria cuatro lotes de descomposición por condición; es decir, 16 lotes en total. Cada lote consistió en 12 bolsas de descomposición, seis bolsas llenas y seis vacías, estas últimas para evaluar el error por acarreo de material al interior de las bolsas (Krishna y Mohan, 2017).

Las bolsas de descomposición se llenaron con $10 \mathrm{~g}$ de muestras compuestas de acículas recién caídas (Costa, 
Pesquero y Moraes, 2019) que fueron previamente colectadas en cada condición. Los lotes se colocaron en enero del 2016, y a los 30, 90, 150, 210, 270 y 365 días de incubación, las bolsas de descomposición ubicadas en cada lote se retiraron de manera aleatoria (una bolsa llena y una vacía).

Las muestras colectadas se secaron en una estufa de circulación forzada de aire a $70{ }^{\circ} \mathrm{C}$ hasta obtener peso constante (aproximadamente a las $72 \mathrm{~h}$ ). La pérdida de peso de la hojarasca de las bolsas de descomposición se determinó para todas las muestras en cada periodo de incubación. A partir de esos valores se estimaron las tasas de descomposición $k$ mediante el modelo de decaimiento exponencial simple negativo (Olson, 1963), cuya fórmula es:

$$
k=-\ln \mathrm{X} t \mathrm{X} 0 \quad k=-\left[\ln \left(\frac{X_{t}}{X_{0}}\right)\right] / t
$$

Donde:

$\mathrm{k}=$ Tasa de descomposición,

$\ln =$ Logaritmo natural,

$\mathrm{X}_{0}=$ peso seco de las acículas al inicio del experimento,

$\mathrm{X}_{\mathrm{t}}=$ peso seco de las acículas en un tiempo determinado, $\mathrm{t}=$ tiempo transcurrido en días.

Los valores de $\mathrm{k}$ se obtuvieron mediante regresión lineal para cada condición a través de un algoritmo elaborado en el software "R" versión 3.2.5 (R Core Team, 2016). Estos correspondieron a tasas de descomposición diarias por lo que se transformaron en valores anuales.

A las muestras de acículas colectadas de los lotes de descomposición se les determinó el contenido de $\mathrm{C}$ y $\mathrm{N}$ para conocer la concentración inicial (en el tiempo 0, antes de su incubación en campo) y después de los diferentes tiempos de incubación en campo. El carbono se analizó con el método de Walkley y Black (1934) para determinar carbono orgánico, mientras que para nitrógeno se hizo el digestado con mezcla diácida y determinado por arrastre de vapor Kjeldahl (Matissek, Schnepel y Steiner, 1998). La liberación de nutrientes $\mathrm{C}$ y $\mathrm{N}$ se calculó a partir de la ecuación propuesta por Guo y Simss (1999):

$$
\mathrm{R}(\%)=\frac{W_{0} C_{0}-W_{t} C_{t}}{W_{0} C_{0}} \times 100
$$

Donde:

$R=$ nutriente liberado,

$W_{0}=$ peso seco inicial,

$W_{t=}$ peso seco remanente de la bolsa de descomposición colectada,

$C_{0}=$ concentración inicial del nutriente $\left(\mathrm{mg} \mathrm{g}^{-1}\right)$,

$C_{t}=$ concentración del nutriente en la hojarasca remanente $\left(\mathrm{mg} \mathrm{g}^{-1}\right)$.

\section{Análisis estadísticos}

Debido a que los datos no presentaron una distribución normal, se aplicó una prueba no paramétrica de KruskalWallis (KW) con 95\% de confiabilidad, bajo la hipótesis de que no hay diferencias estadísticas en la producción de hojarasca y la tasa de descomposición entre condiciones de estudio (R5, R12 y R14) y el sitio de referencia. Posteriormente se realizó una prueba post hoc para comparaciones múltiples con el método de Tukey-Kramer a $95 \%$ de confiabilidad; se consideran diferencias significativas cuando $\mathrm{p} \leq 0.05$ (Sokal y Rohlf, 1995). Los análisis estadísticos se realizaron con el software $\mathrm{R}$ versión 3.2.5 (R Core Team, 2016) y el paquete PMCMR (Pohlert, 2016).

\section{Resultados}

\section{Producción de hojarasca}

Se encontraron diferencias estadísticas altamente significativas en la producción de hojarasca entre las reforestaciones y el sitio de referencia $(\phi \leq 0.000097)$. La prueba post hoc mostró diferencias significativas de producción de hojarasca entre R5 y R12 ( $\mathrm{p} \leq 0.023)$ y entre R5 y R14 ( $\mathrm{p} \leq$ 0.00003 ) (Fig. 2). La producción más alta se presentó en la reforestación de mayor edad (R14), con un total de 0.3099 $\mathrm{kg} \mathrm{m}^{-2}$ año-1, mientras que la menor en la reforestación de 5 años de edad (R5), con menos de 15\% de lo registrado en R14 (0.0436 $\mathrm{kg} \mathrm{m}^{-2}$ año $\left.^{-1}\right)$ (Fig. 2). 
Se observó también una variación temporal en la producción de hojarasca entre las condiciones analizadas. La menor variación se presentó en R5, que para cada mes tuvo la menor producción de hojarasca de todas las condiciones. En el mes de abril se presentaron los picos de producción más altos en todas las condiciones, con excepción también de R5; seguido de junio y julio (Fig. 3).

\section{Tasa de descomposición}

La tasa de descomposición de la hojarasca (acículas) de las especies analizadas mostró que bajo ninguna de las condiciones estudiadas ocurrió la desintegración completa de las muestras durante los primeros 365 días de evaluación. En este periodo, las muestras retuvieron entre $58 \%$ y $75 \%$ de su peso inicial. La tasa de descomposición más alta correspondió a la obtenida en el sitio de referencia (SR) dominado por $P$. cembroides $(k=0.676)$. Las tasas de descomposición en R5, R12 y R14 presentaron poca variación entre sí, con valores de $k=0.353, k=0.265$ y $k$ $=0.229$, respectivamente. El análisis de Kruskal-Wallis mostró diferencias significativas entre las tasas de descomposición de las condiciones estudiadas $(p \leq$ 0.01066). La prueba de Tukey y Kramer confirmó diferencias altamente significativas $(\mathrm{p} \leq 0.0098)$ para las tasas de descomposición entre SR y R14 (Fig. 4).

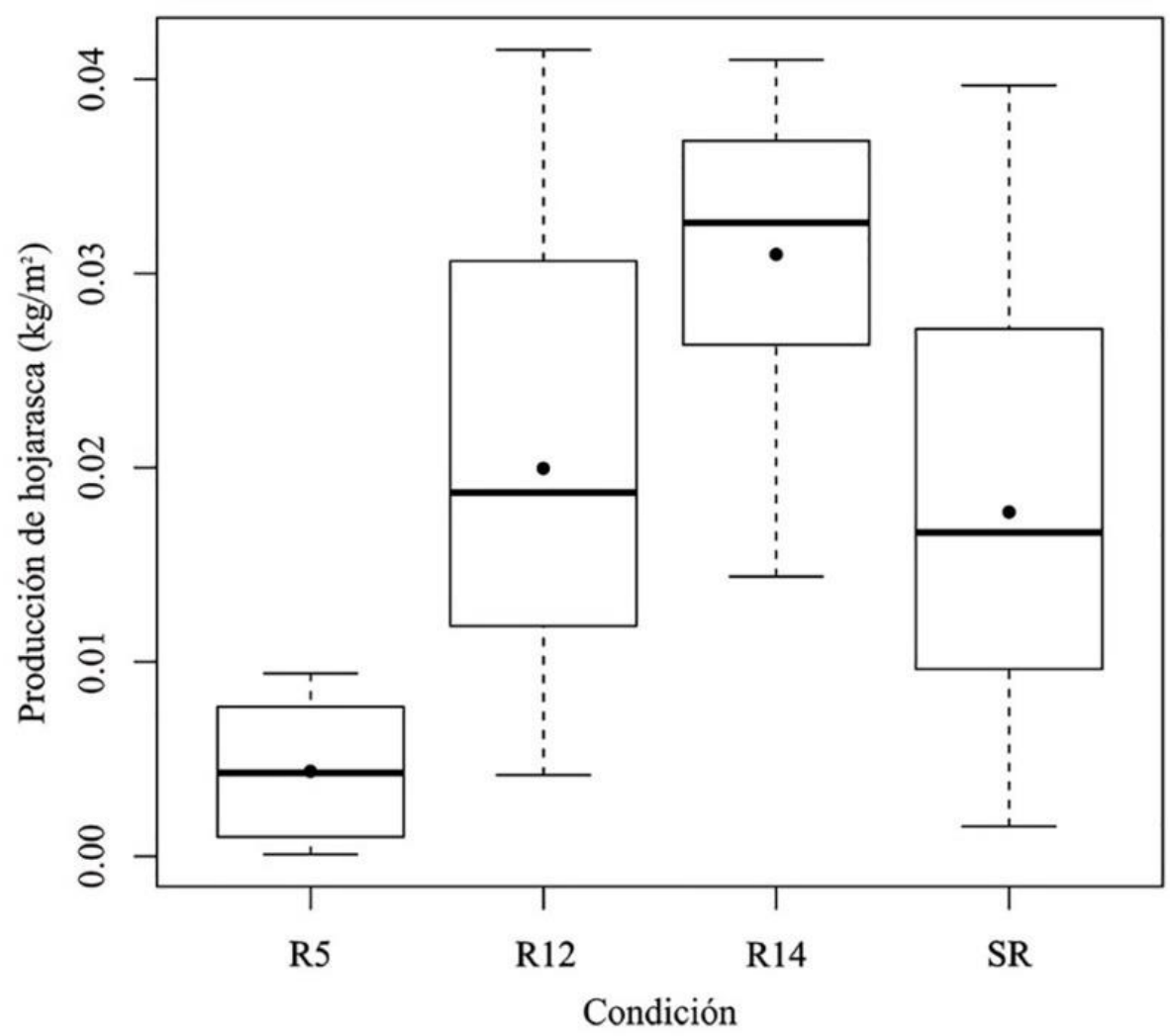

FIgURA 2. Producción anual de hojarasca en las reforestaciones de 5 años, 12 años y 14 años (R5, R12 y R14, respectivamente) y el sitio de referencia (SR) en El Porvenir, Hidalgo. 


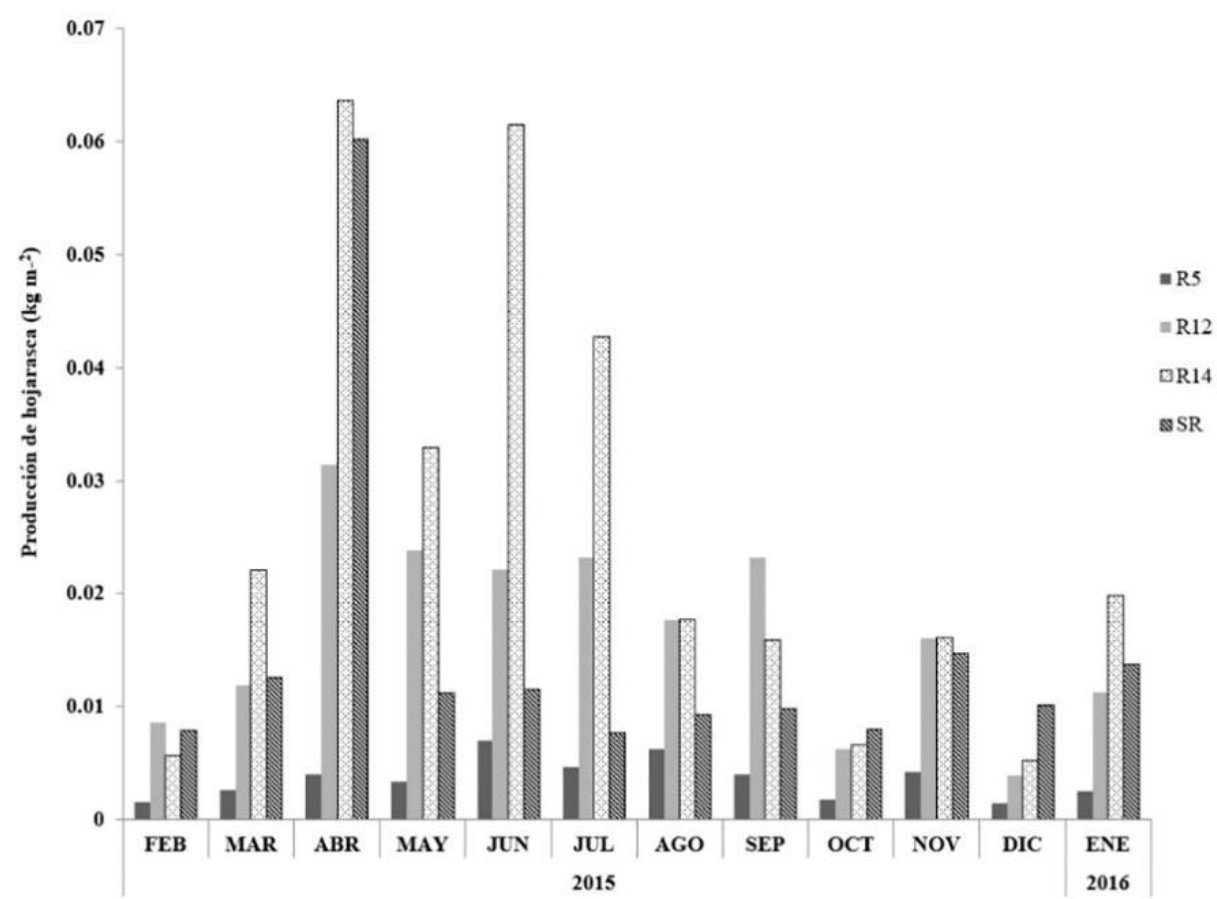

FIGURA 3. Producción mensual de hojarasca (2015-2016) en reforestaciones (R5, R12 y R14) con Pinus greggii y el sitio de referencia (SR) con Pinus cembroides en El Porvenir, Hidalgo.

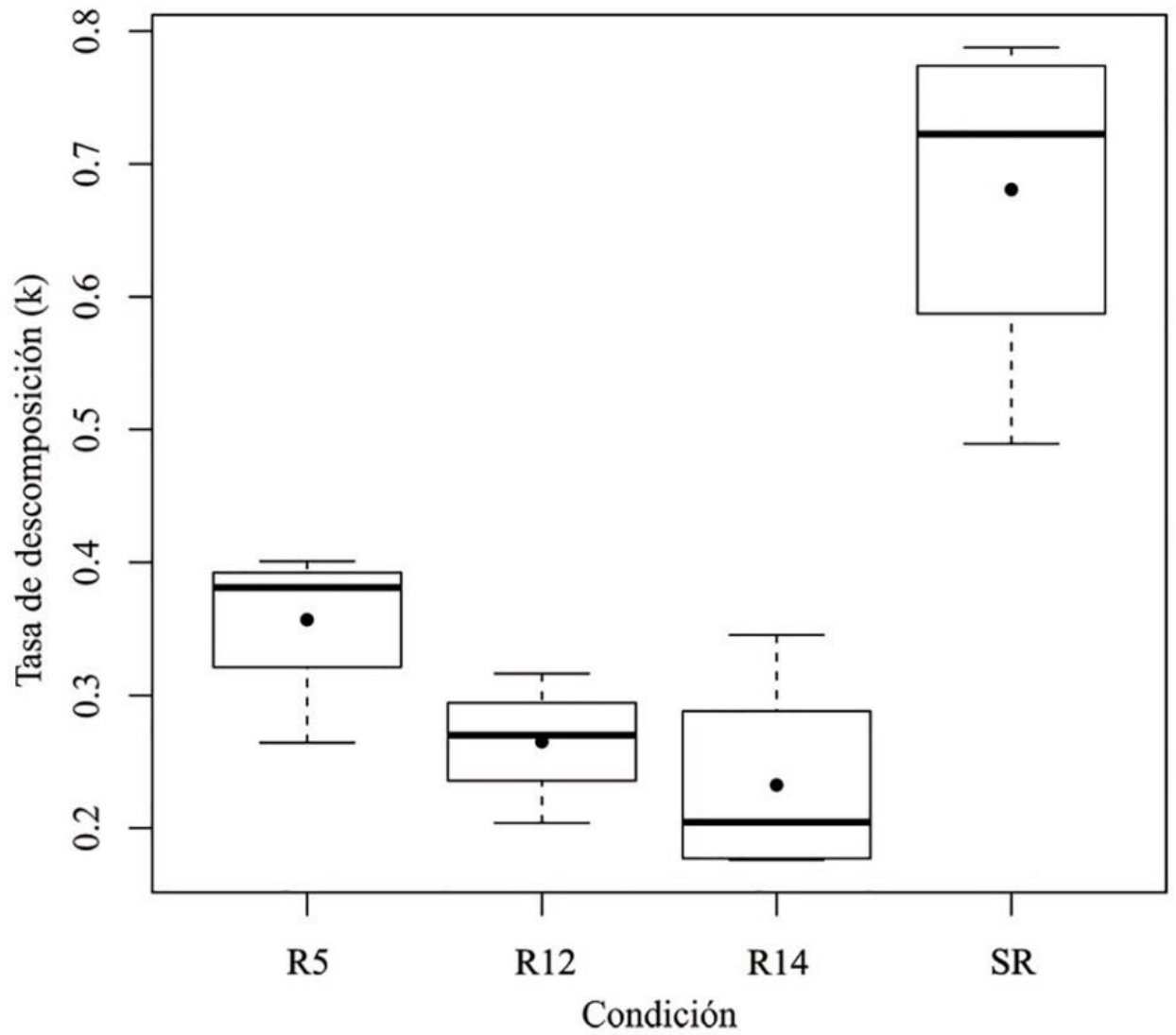

Figura 4. Tasas de descomposición de hojarasca en las reforestaciones de 5 años, 12 años y 14 años (R5, R12 y R14) y el sitio de referencia (SR) en El Porvenir, Hidalgo. 
No se detectó una clara tendencia en el comportamiento independiente de las concentraciones de $\mathrm{C}$ y $\mathrm{N}$ con la edad del rodal después de los distintos tiempos de incubación, a diferencia de la relación C:N, en donde la condición con mayor edad (R14) registró la mayor concentración inicial (Tabla 1). En general, se encontró una disminución en las concentraciones de $\mathrm{C}$ finales con respecto a los valores iniciales, con excepción de los registrados para R12 (Tabla 1). Al final del periodo de incubación (365 días), se registró que la concentración más alta de carbono orgánico se presentó en R12 (49.14\%), mientras la menor en SR (35.88\%) (Tabla 1). Por el contrario, las concentraciones de $\mathrm{N}$ evidenciaron que todos los valores finales son considerablemente mayores a los iniciales, siendo este incremento más notorio en R14. La menor concentración inicial registrada fue en R12 (0.69\%), mientras que la mayor se obtuvo en el sitio de referencia (SR) (1.18\%). El registro más alto obtenido al final del experimento correspondió a R14 (1.53\%), ligeramente superior a SR (1.3\%); mientras que el más bajo fue de $0.89 \%$ para R12 (Tabla 1).

Con respecto a la liberación de $\mathrm{C}$ y $\mathrm{N}$ al ecosistema, se aprecia que SR fue la condición con los valores finales más altos (51.68\% y $36.35 \%$, respectivamente), aun cuando no hay un patrón consistente en el comportamiento por condición y en los diferentes periodos de incubación (Fig.
$5 \mathrm{~A}$ y 5B); con excepción de R5, R14 y SR, en donde la liberación de carbono orgánico tiende a incrementar en el tiempo (Fig. 5A). En R12 se observó retención de C durante los primeros 150 días del período de incubación, por lo que para esta condición se registró la menor liberación de este nutrimento (Fig. 5A). En ambos nutrimentos hubo comportamientos inusuales entre periodos de incubación, siendo R12 un ejemplo atípico en la liberación de carbono (Fig. 5A) y R14 en la de nitrógeno (Fig. 5B).

\section{DISCUSIÓN}

\section{Variabilidad de la producción de hojarasca}

Contrario a la hipótesis planteada, la producción de hojarasca en las cuatro condiciones analizadas presentó variaciones temporales, espaciales y entre especies. La menor producción en R5 puede explicarse por el poco desarrollo estructural del arbolado, mismo que influye directamente en un menor aporte de hojas, ramillas, flores y frutos (Xuluc-Tolosa et al., 2003; Gaspar-Santos et al., 2015), ya que de acuerdo con Montoya-Reyes et al. (2019), la reforestación R 5 es casi 3 veces menor en altura, área basal, diámetro normal y cobertura de copa con respecto a R12 y R14.

TABLA 1. Porcentajes promedio de concentración de carbono $(\mathrm{C})$ y nitrógeno $(\mathrm{N})$ y relación C:N al inicio y fin del periodo de incubación en el sitio de referencia (SR) y en reforestaciones de 5 años, 12 años y 14 años (R5, R12 y R14, respectivamente) en El Porvenir, Hidalgo.

\begin{tabular}{cccccccc}
\hline \multirow{2}{*}{ Condición } & \multicolumn{2}{c}{$\% \mathrm{C}$} & \multicolumn{2}{c}{$\% \mathrm{~N}$} & \multicolumn{2}{c}{ C: } & \\
& Inicial & Final & Inicial & Final & Inicial & Final \\
\hline R5 & 52.26 & 47.58 & 0.93 & 1.06 & 56.19 & 44.89 \\
R12 & 40.56 & 49.14 & 0.69 & 0.89 & 58.78 & 55.21 \\
R14 & 53.04 & 38.61 & 0.77 & 1.53 & 68.88 & 25.23 \\
SR & 42.9 & 35.88 & 1.18 & 1.3 & 36.36 & 27.60 \\
\hline
\end{tabular}


Al considerar la reforestación con mayor edad (R14), los resultados fueron considerablemente mayores a los publicados por Gutiérrez, Méndez, Flores, Ramírez y Gutiérrez (2012) para reforestaciones de P. greggii con 17 años ubicadas en Coahuila, $\mathrm{México}_{s}$ donde se registró una producción de hojarasca de $0.107 \mathrm{~kg} \mathrm{~m}^{-2}$ año-1, en un área que presenta precipitación y temperatura media anual ligeramente menores $\left(521 \mathrm{~mm} \mathrm{y} 13.3^{\circ} \mathrm{C}\right.$ ) a las de $\mathrm{El}$ Porvenir $\left(555.6 \mathrm{~mm}\right.$ y $\left.15.3{ }^{\circ} \mathrm{C}\right)$.

Por su parte, Návar-Cháidez y Jurado-Ybarra (2009) registraron una mayor caída de hojarasca en rodales primarios de Pinus cembroides $\left(0.254 \mathrm{~kg} \mathrm{~m}^{-2}\right.$ año-1) del noreste de México comparados con el sitio de referencia de El Porvenir $\left(0.177 \mathrm{~kg} \mathrm{~m}^{-2}\right.$ año-1), valores que contrastan respecto a reforestaciones llevadas a cabo con la misma especie $\left(0.097 \mathrm{~kg} \mathrm{~m}^{-2}\right.$ año-1) referida por Gutiérrez et al. (2012), aun cuando en este último estudio se tuvo una densidad casi tres veces mayor (2382 árboles ha-1) a la estimada por Montoya-Reyes et al. (2019) para El Porvenir. Si bien la densidad arbórea no había sido considerada un factor determinante en cuanto a la producción de hojarasca (Gunadi, 1994), estudios recientes demuestran lo contrario (Deng y Janssens, 2006; Gutiérrez et al., 2012), y permiten plantear que las diferencias en la producción de hojarasca de Pinus cembroides registradas en SR podrían explicarse parcialmente por este causal.

Rocha-Loredo y Ramírez-Marcial (2009) atribuyen el incremento en la acumulación de hojarasca con la etapa sucesional de un sitio a la mayor producción de biomasa viva y al incremento en la caída de biomasa foliar. Del mismo modo, Wang, Wang, Fan y Yu (2007) concluyen que la caída de hojarasca está estrechamente relacionada con el crecimiento y productividad del bosque. Lo anterior coincide con los hallazgos encontrados en El Porvenir ya que, por un lado, a mayor edad de las reforestaciones se produjo una mayor caída de hojarasca, pero también, esta producción fue mayor en R12 y R14 con respecto a SR; es decir, mayor en reforestaciones con $P$. greggii que con $P$. cembroides, lo cual puede ser explicado en parte debido a la fenología y dinámica de crecimiento de ambas especies.
De acuerdo con Ramírez-Herrera, Vargas-Hernández y López-Upton (2005), P. greggii es uno de los componentes estructurales clave en las comunidades en las que crece, formando parte del dosel dominante, condición que se debe a su mayor tasa de crecimiento (altura, diámetro y cobertura; Gómez-Romero et al., 2012) así como una mayor velocidad de senescencia de las acículas con respecto a $P$. cembroides (Martínez, 1999), especie que ha desarrollado estrategias de supervivencia en ambientes deficitarios de agua y, en consecuencia, tiene un ritmo de crecimiento más lento y presenta follaje perenne (Zavala-Chávez y GarcíaMoya, 1991; Gutiérrez et al., 2012). Esto explicaría el porqué del denso espesor en el piso forestal en los sitios bajo reforestación, a diferencia del sitio de referencia, y también podría precisar las mayores tasas de producción registradas en los mismos.

Por otra parte, de acuerdo con los resultados, la producción de hojarasca también varió en el tiempo, con mayor producción por parte de $P$. greggii a lo largo del año de medición, resultado semejante al registrado por Gutiérrez et al. (2012) en plantaciones forestales de Coahuila con edad similar a R14. De hecho, la máxima producción anual de hojarasca de ambas especies ( $P$. greggii y $P$. cembroides) coincide con estudios diversos llevados a cabo con Pinus pseudostrobus Brongn. (Rocha-Loredo y Ramírez-Marcial, 2009; González-Rodríguez et al., 2011), Pinus oocarpa Schiede ex Schltdl. (Rocha-Loredo y RamírezMarcial, 2009), Pinus cooperi C.E. Blanco (Moreno et al., 2018), P. cembroides (Návar-Cháidez y Jurado-Ybarra, 2009; Gutiérrez et al., 2012; Reyes-Carrera, Méndez-González, Nájera-Luna y Cerano-Paredes, 2013) y P. greggii (Gutiérrez et al., 2012), lo que sugiere que la temporada comprendida entre abril y junio es en la que se registra la mayor productividad para algunas especies mexicanas del género Pinus, independientemente de la especie y la localidad.

De hecho, como lo mencionan Krishna y Mohan (2017), en cualquier tipo de bosque la mayor caída de hojarasca se produce en un momento determinado en el año, por lo que el comportamiento de una especie, o grupo de ellas, está determinado por la ocurrencia de las fases fenológicas como consecuencia de los estímulos de los 
elementos del clima, principalmente la temperatura y la precipitación. Resultados similares han sido generados por Gutiérrez et al. (2012) y Reyes-Carrera et al. (2013) en plantaciones forestales y rodales primarios de P. greggii y $P$. cembroides en Coahuila.

\section{Descomposición y dinámica de la relación C:N}

En cuanto a la tasa de descomposición de las acículas de $P$. cembroides en el sitio de referencia, el coeficiente registrado después de 12 meses es similar al obtenido por RochaLoredo y Ramírez-Marcial (2009) para P. oocarpa en diferentes condiciones sucesionales del bosque de PinoEncino de Chiapas, en donde registraron un valor de $k=$ 0.63. No obstante, estos resultados son mayores a los registrados en las reforestaciones con $P$. greggii en $\mathrm{El}$ Porvenir, situación que puede ser explicada por las concentraciones de celulosa y lignina encontradas por García-Osorio (2017), ya que P. cembroides presentó en promedio $3 \%$ y $2 \%$ menor contenido de estos compuestos. De acuerdo con Chenu et al. (2015), la descomposición es más rápida en hojarasca de menor calidad; esto es, menos lignificada y con mayor contenido nutrimental, como la observada en SR.

Contrario a la hipótesis planteada, el coeficiente de descomposición no tuvo un comportamiento gradual conforme a la edad de reforestación, ya que posterior a SR, el rodal con menor edad (R5) fue el que presentó una mayor tasa de descomposición $(k=0.353)$. Esto puede deberse a la apertura del dosel en este sitio derivada de su condición estructural (García-Osorio, 2017; Montoya-Reyes et al., 2019), escenario que permite una mayor entrada de luz solar, lo que aumenta en consecuencia la temperatura del suelo y, por ende, la velocidad de descomposición de la hojarasca; como lo sugieren algunos autores (Wang et al., 2010; Rubiano-Cardona et al., 2013; Krishna y Mohan, 2017) para otros ecosistemas forestales templados a escala mundial.

De igual forma, con base en la relación C: $\mathrm{N}$ registrada en El Porvenir y tomando de referencia algunos planteamientos teóricos al respecto (Graca et al., 2005;
Karberg et al., 2008; Chenu et al., 2015), es posible explicar la mayor tasa de descomposición que presentó SR. El índice C:N mide la calidad de la hojarasca y permite predecir la velocidad de descomposición de la misma, por lo que si la concentración de $\mathrm{C}$ en la hojarasca es alta y la de $\mathrm{N}$ baja (lo que implica una relación C:N alta), más $\mathrm{N}$ será inmovilizado por la fauna edáfica y habrá menos disponibilidad del mismo para ser asimilado por las plantas (lo que significa una lenta descomposición, como las observadas en R12 y R14). Por el contrario, con una concentración mayor de N el inmovilizado será menor y el disponible para las plantas mayor, como se aprecia en SR (Tabla 1).

Así, aun cuando las concentraciones iniciales de C:N siguen una tendencia de incremento gradual entre edades de reforestación en El Porvenir, las cantidades de lignina y las concentraciones iniciales independientes de $\mathrm{C}$ y $\mathrm{N}$ son los factores determinantes tanto en la producción de hojarasca como en su descomposición, debido a las diferencias en la calidad de la hojarasca y la disponibilidad de nutrimentos en el suelo que forman parte del ciclo. Por lo que, como lo sugieren Krishna y Mohan (2017), la especie bajo análisis debe ser considerada al realizar este tipo de estudios, debido a que restos vegetales con bajas cantidades de lignina y menores concentraciones de C:N presentan una mayor pérdida de masa en menor tiempo, lo que concuerda con los altos porcentajes de liberación de $\mathrm{C}$ y N en SR para P. cembroides.

Con base en los hallazgos generados en la presente investigación y con los aportes obtenidos de otras investigaciones integrales generadas en El Porvenir, Hidalgo, es posible plantear que las áreas bajo estudio se encuentran en un proceso gradual de recuperación a nivel ecosistémico, que va desde grupos biológicos como la mesofauna (García-Osorio, 2017), la producción y descomposición de hojarasca (datos del presente estudio), hasta la composición, estructura y diversidad de la avifauna y su relación ecológica con la vegetación forestal (MontoyaReyes et al., 2019). De manera que, como lo plantean Ventura-Ríos et al. (2017) para el caso particular de El Porvenir, la reforestación con $P$. cembroides y $P$. greggii ha 
resultado en una exitosa estrategia para la rehabilitación de bosques perturbados.

\section{CONCLUSIONES}

La caída de hojarasca presentó variaciones espaciales, temporales y entre especies en El Porvenir, Hidalgo, con una mayor producción en la reforestación de mayor edad (R14), y durante los meses de abril a julio; mientras que la menor producción se presentó en la de 5 años (R5). La tasa de descomposición de hojarasca disminuyó con la edad de la reforestación. Sin embargo, la tasa más alta se presentó en el sitio de referencia (SR). A pesar de que la cantidad de hojarasca producida en SR no difirió de la registrada en la reforestación de mayor edad, la tasa de descomposición $k$ observada sí fue mayor en el primero. Lo anterior implica que, si bien el proceso de incorporación de materia orgánica (hojarasca) se ha recuperado hasta alcanzar cantidades similares al sitio de referencia, las poblaciones de descomponedores aún están en proceso de estructuración; escenario que sugiere una paulatina recuperación integral del ecosistema a medida que incrementa la edad de reforestación.

\section{RECONOCIMIENTOS}

Esta investigación fue financiada por el Programa de Paisajes Sustentables de la Agencia para el Desarrollo Internacional de Estados Unidos de América, a través de la Oficina de Programas Internacionales del Servicio Forestal del Departamento de Agricultura y el Northern Research Station, por medio del proyecto "Reducing greenhouse gas emissions and improving Forest Management in Mexico". Se agradece al Consejo Mexicano de Ciencia y Tecnología (Conacyt) por la beca otorgada al primer autor para sus estudios de Maestría, así como al Colegio de Postgraduados Campus Montecillo por las facilidades prestadas para llevar a cabo la presente investigación. Un extenso agradecimiento a la Comunidad del Porvenir, Santiago de Anaya, Hidalgo, por permitir realizar esta investigación. De igual manera a la SEMARNAT-Hidalgo.Se agradece también a la Dirección General de Asuntos del Personal Académico (DGAPA) de la UNAM por la beca posdoctoral otorgada al quinto autor para colaborar en este proyecto en el análisis de datos.

\section{REFERENCIAS}

Bisht, V. K., Nautiyal, B. P., Kuniyal, C. P., Prasad, P., \& Sundriyal, R. C. (2014). Litter production, decomposition, and nutrient release in subalpine forest communities of the Northwest Himalaya. Journal of Ecosystems, 2014, 294867. doi: 10.1155/2014/294867

Carnevale, N. J., \& Lewis, J. P. (2001). Litterfall and organic matter decomposition in a seasonal forest of the eastern Chaco (Argentina). Revista de Biología Tropical, 49(1), 203-212.

Castellanos-Barliza, J., \& J. D. León P. (2010). Descomposición de hojarasca y liberación de nutrientes en plantaciones de Acacia mangium (Mimosaceae) establecidas en suelos degradados de Colombia. Revista de Biología Tropical, 59(1), 113-128. doi: 10.15517/RBT.V59I1.3182

Chenu, C., Rumpel, C., \& Lehmann, J. (2015). Methods for studying soil organic matter: nature, dynamics, spatial accessibility, and interactions with minerals. En A. P. Eldor (Ed.), Soil microbiology, ecology and biochemistry (4a ed; pp. 383-419). Waltham, Massachusetts: Academic Press.

Coleman, D. C., \& Crossley J. D. A. (2003). Fundamentals of soil ecology. Burlington, Massachusetts: Academic Press.

Comisión Nacional Forestal [Conafor]. (2012). Localidad "El Porvenir" del Ejido el Encino, Modelo de restauración de ecosistemas forestales y de participación social. Boletín Conafor, Gerencia Estatal Hidalgo.

Costa, S., Pesquero, M., \& Moraes, M. (2019). Litterfall deposition and decomposition in an dtlantic forest in Southern Goiás. Floresta e Ambiente, 26(2), e20170744. doi: 10.1590/2179-8087.074417

Deng, Z., \& Janssens, M. J. J. (2006). Litter fall production in westafrican forests and plantations. Documento presentado en Conference on International Agricultural Research for Development. University of Bonn, October 11-13, 2006. Zurich, Switzerland. pp:1-5

Domínguez-Calleros, P. A., Rodríguez-Laguna, R., Capulín-Grande, J., Razo-Zárate R. y Díaz-Vásquez, M. A. (2017). Influencia de factores edáficos en el crecimiento de una plantación de Pinus greggii Engelm. en Santiago de Anaya, Hidalgo, México. Madera y Bosques, 23(2), 145-154. doi: 10.21829/myb.2017.2321522

García-Osorio, M. (2017). Producción y descomposición de hojarasca, y estructura de las comunidades de ácaros oribátidos, en áreas bajo rehabilitación en El Porvenir, Hidalgo. Tesis de Maestría. Postgrado en Ciencias Forestales. Colegio de Postgraduados Campus Montecillo. Texcoco, México. 
García-Velásquez, L. M., Ríos-Quintana, A., \& Molina-Rico, L. J. (2010). Estructura, composición vegetal y descomposición de hojarasca en el suelo, en dos sitios de un bosque nublado andino (reforestado y en sucesión espontánea) en Peñas Blancas, Calarcá (Quindío), Colombia. Actualidades Biológicas, 32(93), 147-164.

Gaspar-Santos, E. S., González-Espinosa, M., Ramírez-Marcial, N., \& Álvarez-Solís, J. D. (2015). Acumulación y descomposición de hojarasca en bosques secundarios del sur de la Sierra Madre de Chiapas, México. Bosque, 36(3), 467-480. doi: 10.4067/S071792002015000300013

Gómez-Romero, M., Soto-Correa, J. C., Blanco-García, J. A., SáenzRomero, C., Villegas, J., \& Linding-Cisneros, R. (2012). Estudio de especies de pino para restauración de sitios degradados. Agrociencia, 46(8), 795-807.

González-Rodríguez, H., Domínguez-Gómez, T. G., Cantú-Silva, I., Gómez-Meza, M. V., Ramírez-Lozano, R. G., Pando-Moreno, M., \& Fernández, C. J. (2011). Litterfall deposition and leaf litter nutrient return in different locations at Northeastern Mexico. Plant Ecology, 212, 1747-1757. doi: 10.1007/s11258-011-9952-9

Graca, M. A. S., Barlocher, F., \& Gessner, M. O. (2005). Methods to study litter decomposition a practical guide. Netherlands: Springer. doi: 10.1007/1-4020-3466-0

Gunadi, B. (1994). Litterfall, litter turnover and soil respiration in two pine forest plantations in Central Java, Indonesia. Journal of Tropical forest Science, 6(3), 310-322.

Guo, L. B., \& Simss, R. E. H. (1999). Litter decomposition and nutrient release via litter decomposition in New Zealand eucalypt short rotation forests. Agriculture, Ecosystems \& Environment, 75, 133-140. doi: 10.1016/S0167-8809(99)00069-9

Gutiérrez, V. M. H., Méndez, G. J., Flores, L. C., Ramírez, D. J. A., \& Gutiérrez, V. B. N. (2012). Caída de hojarasca en plantaciones de Pinus greggii Engelm. y Pinus cembroides Zucc., en Coahuila, México. Revista Fitotecnia Mexicana, 35(2), 123-133.

Hanpattanakit, P. \& Chidthaisong, A. (2012). Litter production and decomposition in dry dipterocarp forest and their responses to climatic factors. GMS ARN International Journal, 6(2012), 169-174.

Karberg, N. J., Scott, N. A., \& Giardina, C. P. (2008). Methods for estimating litter decomposition. En Hoover, C. M. (Ed.), Field measurements for forest carbon monitoring (pp. 103-111). New York: Springer. doi: 10.1007/978-1-4020-8506-2_8

Krishna, M. P., \& Mohan, M. (2017). Litter decomposition in forest ecosystems: a review. Energy, Ecology and Environment, 2(4), 236249. doi: https://doi.org/10.1007/s40974-017-0064-9

López-Hernández, J. M., González-Rodríguez, H., Ramírez-Lozano, R. G., Cantú-Silva, I., Gómez-Meza, M. V., Pando-Moreno, M., \&
Estrada-Castillón, A. E. (2013). Producción de hojarasca y retorno potencial de nutrientes en tres sitios del estado de Nuevo León, México. Polibotánica, 35, 41-64.

Martínez, G. (1999). Estado del conocimiento de Pinus greggii Engelm. Tesis de Licenciatura. División de Ciencias Forestales. Universidad Autónoma Chapingo. Texcoco, México.

Matissek, R., Schnepel, F., \& Steiner, G. (1998). Análisis de los alimentos: fundamentos, métodos y aplicaciones. Zaragoza, España: Editorial Acribia S.A.

Montoya-Reyes, F., Plascencia-Escalante, F. O., Ugalde-Lezama, S., Tarango-Arámbula, L. A., García-Osorio, M. T., \& BeltránRodríguez, L. A. (2019). Forest rehabilitation and its role in bird conservation in El Porvenir, Hidalgo, Mexico. Revista Chapingo Serie Ciencias Forestales y del Ambiente, 25(3), 441-459. doi: 10.5154/r.rchscfa.2018.12.091

Moreno, M., Domínguez, T., Alvarado, Ma., Colín, J., Corral, S., \& González, H. (2018). Contribution and decomposition of leaf litter in temperate forests of the El Salto region, Durango. Revista Mexicana de Ciencias Forestales, 9(47), 70-93. doi: https://doi.org/10.29298/rmcf.v9i47.180

Muoghalu, J. I. \& Odiwe, A. I. (2011). Litter production and decomposition in cacao (Theobroma cacao) and kolanut (Cola nitida) plantations. Ecotropica, 17, 79-90.

Návar-Cháidez, J. J. \& Jurado-Ybarra, E. (2009). Productividad foliar y radicular en ecosistemas forestales del noreste de México. Revista Mexicana de Ciencias Forestales, 34(106), 89-106.

Olson, J. S. (1963). Energy Storage and the balance of producers and decomposers in ecological systems. Ecology, 44(2), 322-331. doi: $10.2307 / 1932179$

Pohlert, T. (2016). The pairwise multiple comparison of mean ranks package (PMCMR). R package version 4.1.

Quinto, M. H., Ramos, P. Y. A., \& Abadía, B. D. (2007). Cuantificación de la caída de hojarasca como medida de la productividad primaria neta en un bosque pluvial tropical en Salero, Chocó, Colombia. Biodiversidad, 26(1), 28-41. doi: 10.18636/biodesarrollo.v26i1.464

R Core Team. (2016). R: A language and environment for statistical computing. R Foundation for Statistical Computing, Vienna, Austria. http:/ /www.R-project.org/

Ramírez-Herrera, C., Vargas-Hernández, J., \& López-Upton, J. (2005). Distribución y conservación de las poblaciones naturales de Pinus greggii. Acta Botanica Mexicana, 72, 1-16. doi: $10.21829 / \mathrm{abm} 72.2005 .997$

Reyes-Carrera, S., Méndez-González, J., Nájera-Luna, J., \& CeranoParedes, J. (2013). Producción de hojarasca en un rodal de Pinus 
cembroides Zucc. en Arteaga, Coahuila, México y su relación con las variables climáticas. Revista Chapingo Serie Ciencias Forestales y del Ambiente, 19(1), 147-155. doi: 10.5154/r.rchscfa.2012.01.006.

Riviera, V. R., Soto, P. L., Núñez, C. C. A., De Jung, B., Hernández, R. Ma. G., \& Ordóñez, D. J. A. B. (2013). Producción y tasa de descomposición de hojarasca en acahuales de selva caducifolia en Chiapas. Revista Mexicana de Ciencias Forestales, 4(20), 20-30. doi: 10.29298/rmcf.v4i20.367

Rocha-Loredo, A. G. \& Ramírez-Marcial, N. (2009). Producción y descomposición de hojarasca en diferentes condiciones sucesionales del bosque de pino-encino en Chiapas, México. Boletin de la Sociedad Botánica de México, 84, 1-12. doi: $10.17129 /$ botsci.2287

Rubiano-Cardona, K., Arcila-Cardona, L. F., Jiménez-Carmona, E., \& Armbrecht, I. (2013). Production, accumulation, and decomposition of leaf litter in a colombian subandean forest and neighboring areas of restoration. Boletin Cientifico. Centro de Museos. Museo de Historia Natural, 17(2), 47-59.

Servicio Meteorológico Nacional. (2019). Normales Climatológicas del estado de Hidalgo, Claves 13109 y 13151, para el periodo 1951 2010. Comisión Nacional del Agua. Recuperado de https://smn.cna.gob.mx/es/informacion-climatologica-porestado? estado $=$ hgo

Sokal, R. R. \& Rohlf, F. J. (1995). Biometry: the principles and practice of statistic in biological research. New York: W. H. Freeman.

Triadiati., Tjitrosemito, S., Guhardja, E., Sudarsono., Qayim, I., \& Leuschner, C. (2011). Litterfall Production and Leaf-litter Decomposition at Natural Forest and Cacao Agroforestry in Central Sulawesi, Indonesia. Asian Journal of Biological Sciences, 4, 221-234. doi: 10.3923/ajbs.2011.221.234

Ventura-Ríos, A., Plascencia-Escalante, F. O., Hernández de la Rosa, P., Ángeles-Pérez, G., \& Aldrete, A. (2017). ¿Es la reforestación una estrategia para la rehabilitación de bosques de pino? Una experiencia en el centro de México. Bosque, 38(1), 55-66. doi: 10.4067/S0717-92002017000100007

Walkley, A. \& Black, A. (1934). An examination of the Degtjareff method for determining soil organic matter, and proposed modification of the chromic acid titration method. Soil Sciences, 37 , $29-38$.

Wang, Q., Wang, S., Fan, B., \& Yu, X. (2007). Litter production, leaf litter decomposition and nutrient return in Cunninghamia lanceolata plantations in south China: effect of planting conifers with broadleaved species. Plant and Soil, 297, 201-211. doi: 10.1007/s11104-007-9333-2
Wang, Q., Wang, S., Xu, G., \& Fan, B. (2010). Conversion of secondary broadleaved forest into Chinese fir plantation alters litter production and potential nutrient returns. Plant Ecology, 209(2), 269-278. doi: https://doi.org/10.1007/s11258-010-9719-8

Xuluc-Tolosa, F. J., Vestera, H. F. M., Ramírez-Marcial, N., CastellanosAlbores, J., \& Lawrence, D. (2003). Leaf litter decomposition of tree species in three successional phases of tropical dry secondary forest in Campeche, Mexico. Forest Ecology and Management, 174, 401-412. doi: 10.1016/S0378-1127(02)00059-2

Yang, Y. S., Guo, J. F, Chen, G. S., Xie, J. S., Cai, L. P., \& Lin, P. (2004). Litterfall, nutrient return, and leaf-litter decomposition in four plantations compared with a natural forest in subtropical China. Annals of Forest Science, 61(5), 465-476. doi: 10.1051/forest:2004040

Zavala-Chávez, F. \& García-Moya, E. (1991). Fenología y crecimiento del brote anual de Pinus cembroides Zucc. de San Luis Potosí, México. Biotam, 3(2), 5-19.

Zhou, G., Guan, L., Wei, X., Zhang, D., Zhang, Q., Yan, J., Wen, D., Lui, J., Liu, S., Huang, Z., Kong, G., Mo, J., \& Yu, Q. (2007). Litterfall production along successional and altitudinal gradients of subtropical monsoon evergreen broadleaved forests in Guangdong, China. Plant Ecology, 188, 77-89. doi: 10.1007/s11258-006-9149-9

\section{Manuscrito recibido el 11 de enero de 2020}

Aceptado el 25 de marzo de 2020

Publicado el 13 de noviembre de 2020

\section{Este documento se debe citar como:}

García-Osorio, M. T., Plascencia-Escalante, F. O., ÁngelesPérez, G., Montoya-Reyes, F., \& Beltrán-Rodríguez, L. (2020). Producción y tasa de descomposición de hojarasca en áreas bajo rehabilitación en El Porvenir, Hidalgo, México. Madera y Bosques, 26(3), e2632099. doi: 10.21829/myb.2020.2632099 Commons Atribución-NoComercialCompartirlgual 4.0 Internacional. 